\title{
1 Capturing scientific knowledge in computable form
}

2

3

4 Jeffrey V. Wong ${ }^{1}$, Max Franz ${ }^{1}$, Metin Can Siper ${ }^{2}$, Dylan Fong ${ }^{1}$, Funda Durupinar ${ }^{3}$, Christian

5 Dallago ${ }^{5,6,7}$, Augustin Luna ${ }^{4,5,8}$, John Giorgi ${ }^{1}$, Igor Rodchenkov ${ }^{1}$, Özgün Babur ${ }^{3}$, John A.

6 Bachman $^{9}$, Benjamin M. Gyori ${ }^{9}$, Emek Demir ${ }^{2, *}$, Gary D. Bader ${ }^{1,10,11 *}$ and Chris Sander ${ }^{4,5,8 *}$

$8{ }^{1}$ The Donnelly Centre, University of Toronto, Toronto, Ontario, M5S 3E1, Canada

92 Computational Biology Program, Oregon Health and Science University, Portland, OR

10 97239, USA

$11^{3}$ Computer Science Department, University of Massachusetts Boston, 100 Morrissey

12 Boulevard Boston, MA 02125

$13{ }^{4}$ Department of Data Sciences, Dana-Farber Cancer Institute, Boston, MA, 02215, USA

$14{ }^{5}$ Department of Cell Biology, Harvard Medical School, Boston, MA, 02215, USA

$15{ }^{6}$ Department of Systems Biology, Harvard Medical School, Boston, MA, 02215, USA

$16{ }^{7}$ Department of Informatics, Technische Universität München, 85748 Garching, Germany

$17{ }^{8}$ Broad Institute of MIT and Harvard, Boston, MA, 02142, USA

$18{ }^{9}$ Laboratory of Systems Pharmacology, Harvard Medical School, Boston, MA, 02115, USA

$19{ }^{10}$ Department of Computer Science, University of Toronto, Ontario, M5S 2E4, Canada

$20{ }^{11}$ Department of Molecular Genetics, University of Toronto, Ontario, M5S 1A8, Canada

$22{ }^{*}$ Correspondence to info@biofactoid.org reaches the principal authors.

24 Availability: Biofactoid server at https://biofactoid.org

25 Keywords: pathway analysis; curation tool; knowledge base 


\section{ABSTRACT}

29 Technological advances in computing provide major opportunities to complement human 30 reasoning and to dramatically speed up science - but only if structured knowledge is 31 available to enable efficient communication between humans and computers. Traditionally, 32 biological knowledge is captured in publications and knowledge bases. Knowledge in papers 33 is not directly in a computable, structured form; curated structured knowledge bases are 34 limited by manual curation processes. To accelerate knowledge capture and communication 35 and keep pace with the rapid growth of scientific reports, we developed the Biofactoid 36 (biofactoid.org) software suite. Biofactoid accelerates the transfer of knowledge from the 37 minds of authors into computable, widely shared, structured knowledge and can be used as 38 part of the standard publication process. Biofactoid is a web-based system for scientists to 39 compose a structured representation of networks of interactions between genes, their 40 products, and chemical compounds, represented using the expressive power of a formal 41 ontology (BioPAX). The resulting knowledge items are shared via public information 42 resources and can be discovered and analyzed in the context of all existing computable 43 knowledge. We envision adoption of software technology for knowledge capture by scientists 44 and publishers as part of an ecosystem of tools, in which scientific reasoning is supported by 45 efficient knowledge computation.

46

47

\section{INTRODUCTION}

Biological pathways organize sets of molecular interactions and reactions that underlie cellular processes and are used for experimental design, interpretation of genomics 51 data (Khatri et al., 2012), understanding of disease mechanisms (Chinen et al., 2016; Santos 52 et al., 2014), and identification of therapeutic targets (Mack et al., 2014). To manage, 53 visualize and interpret the large amount of available pathway information, researchers 54 require computational tools such as pathway information resources, structured data 55 representation standards, and analysis software (Demir et al., 2010; Franz et al., 2016; 56 Jassal et al., 2020; Pratt et al., 2015; Rodchenkov et al., 2020; Shannon et al., 2003). These

57 computational tools are capable of accessing increasing amounts of pathway and interaction 58 data (Bader et al., 2006) collected through centralized (Gene Ontology Consortium, 2015; 59 Jassal et al., 2020) or crowdsourced curation efforts (Slenter et al., 2018). Nevertheless, 60 these existing efforts are not able to achieve wide coverage over the rapidly growing 61 scientific corpus (over 1.3 million new PubMed articles/year) (Bornmann and Mutz, 2015; 62 Cordero et al., 2016), arguing for the development of scalable and sustainable approaches 63 to complement existing approaches to knowledge acquisition (Attwood et al., 2015; Imker, 64 2018).

65 For reasons of efficiency and accuracy, structured knowledge of biomedical 66 discoveries could be provided directly by the original authors of research reports, rather than 67 captured indirectly by subsequent reading of the reports by knowledge base teams. By 68 analogy, the molecular 3D structures submitted directly by authors to the Protein DataBank 69 (PDB) (Berman et al., 2000) have become a key resource among structural biologists, so 70 much so that publication in major journals is contingent on data deposition. Similar 71 requirements and community practice have also led to direct submission of DNA sequence 72 and transcriptomics information to public databases as core components of the biological 73 research information infrastructure. In contrast, there are few efforts and little technology to 
74 support direct submission by authors of biological pathway information and related 75 knowledge in computable form.

Here we describe Biofactoid (biofactoid.org), a web-based software system that empowers authors to capture and share structured human- and machine-readable summaries of molecular-level interactions described in their publications. Without such a curation support tool, authors would have to spend unnecessary time figuring out the computational form of their knowledge for incorporation into a computation-enabled knowledge base. To overcome this and other significant barriers to computable knowledge acquisition and sharing, Biofactoid was developed to ease pathway curation and to rapidly generate expressive, structured representations with minimal user training. Structured knowledge newly acquired in this way becomes part of the global pool of pathway knowledge and can be shared in resources such as Pathway Commons (Rodchenkov et al., 2020), Network Data Exchange (NDEx) (Pratt et al., 2015), and STRING (Szklarczyk et al., 2017), to enhance information discovery and analysis. Biofactoid can be used by authors and publishers to share structured information from existing and new research articles, in voluntary or required mode. The development of Biofactoid and related computational tools helps support human-computer communication and inference algorithms in an ecosystem in which scientific reasoning is increasingly assisted by broad and deep knowledge computation tools.

\section{RESULTS}

\section{Data sharing workflow}

Biofactoid enables molecular-level detail of biological processes reported in peer-reviewed publications to be shared in a structured format accessible to humans and computers. Interactions (including binding, post-translational modification, and transcription/translation) involving molecules of various types (proteins, nucleic acids, genes, or chemicals, e.g., metabolites and drug compounds) can be represented. Users begin by entering the article title, or identifier (e.g. PubMed identifier or Digital Object Identifier, DOI). Article metadata (authors, abstract \& journal issue) is automatically imported if available. Next, users draw a network of biological entities and interactions using the Biofactoid curation tool, which is akin to graphical illustration software (e.g. Microsoft Powerpoint, Adobe Illustrator), with the added ability to embed structured information (such as database links and molecule types) about the network components and interaction types. The major features of the Biofactoid curation tool are as follows:

1. Molecular entities. Genes, gene products or chemicals are created in the network using an "add entity" tool, which creates a node (circle shape) that the user labels (Figure 1A). Biofactoid automatically maps the label to an external database record, NCBI Gene for genes and their products, and ChEBI for small molecules (Brown et al., 2015; Hastings et al., 2016). This mapping represents the top hit of a search based on the similarity between a node label and a database record's list of synonyms; for genes, organisms are given priority based upon the organism of genes previously added to the network. Users can update the mapping by selecting another organism (e.g. human p53 or mouse p53) or product type (i.e., RNA or protein). Alternatively, users may assign an alternate and distinct database entry from an annotated list of options. 
2. Interactions. A "draw interaction" tool lets users link two nodes by clicking or tapping and dragging (Figure 1B). Biofactoid uses common visual representations in biological process diagrams (a normal arrow indicates stimulation or activation, a 'T-bar' arrow head indicates inhibition or repression, and an undirected line indicates any other interaction) and generally follows an Activity Flow representation paradigm (Le Novère et al., 2009). The relationship type can be refined by selecting from a list that currently includes binding, transcription/translation, or common forms of post-translational modifications, with more detailed interaction types following the BioPAX ontology (Demir et al., 2010) to be added in a future release.

3. Complexes. Molecular complexes can be created by dragging gene or chemical nodes together (Figure 1B). Those nodes are then enclosed by a box that represents the complex, which can be named.

4. Automatic saving and co-editing. Pathway representations in Biofactoid are automatically saved as changes are made and a live-sync capability enables multiple authors to collaboratively edit the same pathway, analogous to Google Docs.

Once complete, the pathway data is validated by pressing a 'Submit' button. At this point, the user may address any potential quality issues (e.g. unlabelled nodes, empty document) and then can confirm submission. Upon submission, Biofactoid makes the shared pathway visible and explorable on the Biofactoid website, posts a graphical abstract to social media (e.g. Twitter: @biofactoid), and makes the pathway data available in standard formats (e.g. BioPAX) to be integrated in public, searchable databases, such as Pathway Commons (Rodchenkov et al., 2020), NDEx (Pratt et al., 2015) and Reactome (Jassal et al., 2020). Biofactoid information thus becomes part of a growing corpus of widely available pathway information in computable form, enhancing both human and computational discovery processes.

\section{Data exploration workflow}

All user-submitted pathways are made immediately available as graphical abstracts on the Biofactoid homepage which researchers, or journal editors tracking a submission, may freely browse. Each pathway is linked to its associated author and article information and its structured data in the Biofactoid 'Explorer' app (Figure 1C). Articles with related content (e.g. molecules and interactions from the Biofactoid pathway) are shown to help the user explore similar knowledge in the literature. This information is accessed via a customized search process from pathway and interaction databases and biomedical literature using natural language processing (NLP) tools and databases (e.g. REACH, INDRA) (Gyori et al., 2017; Valenzuela-Escárcega et al., 2018), with results ranked by similarity to text associated with the query pathway, using a deep-learning based method (Giorgi et al., 2020). A future release will link Biofactoid data to similar pathways, accessed from pathway knowledge bases.

\section{DISCUSSION}

Biofactoid has focused on the capture of published pathway information to augment discovery, attribution and communication of scientific knowledge. In developing our strategy, we have considered how technology can best be used to aid authors; the result is a generic approach that can be extended towards the acquisition of other types of biological knowledge in computable form. A convenient time to capture knowledge is during 
166 publication, as this represents a period when authors provide other types of data for 167 deposition (e.g. sequence data) and is a time when they are most keenly aware of the 168 information used in their report. Thus, Biofactoid will ideally be part of the publication 169 process, but also possibly as part of more general crowdsourcing efforts, such as curating 170 and sharing all published knowledge about SARS-CoV-2 (Ostaszewski et al., 2020). To be 171 successful, Biofactoid development has focused on exploring key elements of efficiency, 172 incentives, added value for researchers and better technology for human-computer 173 communication. The computable knowledge capture model proposed here includes formal 174 knowledge representation using ontologies, easy to use curation support software that links 175 molecules to corresponding database identifiers (grounding or normalization) and a 176 connection with the publication process and author attribution. This model expands on prior 177 work defining digital abstracts and building pathway data crowdsourcing efforts and software 178 (Bharadwaj et al., 2017; Ceol et al., 2008; Liechti et al., 2017; Pratt et al., 2015; Seringhaus 179 and Gerstein, 2007; Slenter et al., 2018; Todorov et al., 2019; Waagmeester et al., 2020). 180 Future development will include expanding the model to capture increasing amounts of 181 literature-described pathway information, such as additional relationship types, context and 182 direct vs. indirect interactions, prioritized by frequency of occurrence in publications.

To improve the efficiency and utility of Biofactoid, we are developing natural language 184 processing (NLP) technology to assist in the Biofactoid workflow and enable the 185 representation of pathways in textual form (Giorgi et al., 2019; Giorgi and Bader, 2020, 2018; 186 Valenzuela-Escárcega et al., 2018). As images and text are the main modes of 187 communication with humans, the system will permit graphical or textual entry of pathway 188 information and automated conversion between these two forms. Automated NLP 189 processing of a manuscript will extract phrases describing pathways (Gyori et al., 2017), 190 which will then be suggested to the author who can choose to include them in their pathway. 191 Further development of NLP methods for the reliable extraction of pathway information from 192 the entire full-text corpus of publications, followed by new tools for curation and quality 193 control, will help get the community closer to the goal of full coverage of current pathway 194 knowledge in computable form. This would significantly increase the benefit to the research 195 community from using powerful methods for network and pathway searches, logical 196 inference of information propagation through pathways, and machine learning for the 197 elucidation of essential cell biological processes, which benefit from increased coverage of 198 network data to find important relationships. We are also improving the search and 199 exploration functions of the system, ensuring Biofactoid information is well connected to 200 other useful knowledge, and that these connections to related information are browsable 201 starting from a Biofactoid entry of interest. In the future, we envision that an author will signal 202 their interests in specific molecules, relationships and context by entering information they 203 publish about these in Biofactoid, which will then act as a custom query that regularly alerts 204 authors to new information (e.g. from publications) related to their interests, including 205 interacting molecules, phenotypes and other biological concepts. This work sets the stage 206 for the development of new information technologies that will help researchers overcome 207 information overload, caused by rapid growth of the scientific literature, and help increase 208 the speed of scientific discovery.

\section{MATERIALS AND METHODS}




\section{Implementation}

Biofactoid is written in JavaScript. The backend server uses a microservice

214 architecture, with Node.js, Express, and RethinkDB. Client-server data synchronization,

215 supporting automatic saving and concurrent editing, uses websockets and a model similar to

216 differential synchronization (Fraser, 2009). The front end uses React and Cytoscape.js

217 (Franz et al., 2016), for network drawing and is optimized for desktop and mobile devices.

218 An administrative dashboard, as well as user curation workflow automation features (e.g.

219 automatic email generation) are integrated into the Biofactoid system to aid system

220 scalability.

221

\section{Availability}

\section{REFERENCES} improve our software.

\section{FUNDING}

None declared.

Biofactoid is available to biomedical researchers for data sharing and exploration via the https://biofactoid.org website. To support bioinformaticians and software developers, all user-contributed pathway data is openly accessible in multiple standard formats, including raw data in JavaScript Object Notation (JSON), the Systems Biology Graphical Notation Markup Language (SBGNML) pathway visualization format (Process Description visual language) (Le Novère et al., 2009; van lersel et al., 2012), and the Biological Pathway Exchange (BioPAX) language (Demir et al., 2010). All source code, documentation and data are open source and freely available through GitHub (github.com/PathwayCommons/factoid), and containerized components are freely available on DockerHub (hub.docker.com/r/pathwaycommons/factoid) enabling others to build on and

Biofactoid development was funded by the US National Institutes of Health (NIH) [U41 HG006623 and U41 HG003751] and the DARPA Big Mechanism and Communicating with Computers programs [ARO W911NF-14-C-0119, W911NF-15-1-0544].

\section{ACKNOWLEDGEMENTS}

We thank Quincey Justman, Miao-Chih Tsai and Anita DeWaard for feedback on making Biofactoid useful for editors and authors; the Reactome database team for support and feedback on the curation workflow; early support from Alfonso Valencia and Miguel Vazquez; finally, the many community members in Toronto, Boston, Portland and beyond who provided feedback on Biofactoid's design and concept.

\section{COMPETING INTERESTS}

Attwood TK, Agit B, Ellis LBM. 2015. Longevity of Biological Databases. EMBnet.journal 21. doi:10.14806/ej.21.0.803

Bader GD, Cary MP, Sander C. 2006. Pathguide: a pathway resource list. Nucleic Acids Res 34:D504-506. doi:10.1093/nar/gkj126

Berman HM, Westbrook J, Feng Z, Gilliland G, Bhat TN, Weissig H, Shindyalov IN, Bourne PE. 2000. The Protein Data Bank. Nucleic Acids Res 28:235-242. doi:10.1093/nar/28.1.235 
Bharadwaj A, Singh DP, Ritz A, Tegge AN, Poirel CL, Kraikivski P, Adames N, Luther K, Kale SD, Peccoud J, Tyson JJ, Murali TM. 2017. GraphSpace: stimulating interdisciplinary collaborations in network biology. Bioinforma Oxf Eng/ 33:3134-3136. doi:10.1093/bioinformatics/btx382

Bornmann L, Mutz R. 2015. Growth rates of modern science: A bibliometric analysis based on the number of publications and cited references: Growth Rates of Modern Science: A Bibliometric Analysis Based on the Number of Publications and Cited References. J Assoc Inf Sci Technol 66:2215-2222. doi:10.1002/asi.23329

Brown GR, Hem V, Katz KS, Ovetsky M, Wallin C, Ermolaeva O, Tolstoy I, Tatusova T, Pruitt KD, Maglott DR, Murphy TD. 2015. Gene: a gene-centered information resource at NCBI. Nucleic Acids Res 43:D36-42. doi:10.1093/nar/gku1055

Ceol A, Chatr-Aryamontri A, Licata L, Cesareni G. 2008. Linking entries in protein interaction database to structured text: the FEBS Letters experiment. FEBS Lett 582:1171-1177. doi:10.1016/j.febslet.2008.02.071

Chinen T, Kannan AK, Levine AG, Fan X, Klein U, Zheng Y, Gasteiger G, Feng Y, Fontenot JD, Rudensky AY. 2016. An essential role for the IL-2 receptor in Treg cell function. Nat Immunol 17:1322-1333. doi:10.1038/ni.3540

Cordero RJB, de León-Rodriguez CM, Alvarado-Torres JK, Rodriguez AR, Casadevall A. 2016. Life Science's Average Publishable Unit (APU) Has Increased over the Past Two Decades. PLOS ONE 11:e0156983. doi:10.1371/journal.pone.0156983

Demir E, Cary MP, Paley S, Fukuda K, Lemer C, Vastrik I, Wu G, D'Eustachio P, Schaefer C, Luciano J, Schacherer F, Martinez-Flores I, Hu Z, Jimenez-Jacinto V, Joshi-Tope G, Kandasamy K, Lopez-Fuentes AC, Mi H, Pichler E, Rodchenkov I, Splendiani A, Tkachev S, Zucker J, Gopinath G, Rajasimha H, Ramakrishnan R, Shah I, Syed M, Anwar N, Babur O, Blinov M, Brauner E, Corwin D, Donaldson S, Gibbons F, Goldberg R, Hornbeck P, Luna A, Murray-Rust P, Neumann E, Ruebenacker O, Reubenacker O, Samwald M, van lersel M, Wimalaratne S, Allen K, Braun B, Whirl-Carrillo M, Cheung K-H, Dahlquist K, Finney A, Gillespie M, Glass E, Gong L, Haw R, Honig M, Hubaut O, Kane D, Krupa S, Kutmon M, Leonard J, Marks D, Merberg D, Petri V, Pico A, Ravenscroft D, Ren L, Shah N, Sunshine M, Tang R, Whaley R, Letovksy S, Buetow KH, Rzhetsky A, Schachter V, Sobral BS, Dogrusoz U, McWeeney S, Aladjem M, Birney E, Collado-Vides J, Goto S, Hucka M, Le Novère N, Maltsev N, Pandey A, Thomas P, Wingender E, Karp PD, Sander C, Bader GD. 2010. The BioPAX community standard for pathway data sharing. Nat Biotechnol 28:935-942. doi:10.1038/nbt.1666

Franz M, Lopes CT, Huck G, Dong Y, Sumer O, Bader GD. 2016. Cytoscape.js: a graph theory library for visualisation and analysis. Bioinforma Oxf Engl 32:309-311. doi:10.1093/bioinformatics/btv557

Fraser N. 2009. Differential synchronizationProceedings of the 9th ACM Symposium on Document Engineering - DocEng '09. Presented at the the 9th ACM symposium. Munich, Germany: ACM Press. p. 13. doi:10.1145/1600193.1600198

Gene Ontology Consortium. 2015. Gene Ontology Consortium: going forward. Nucleic Acids Res 43:D1049-1056. doi:10.1093/nar/gku1179

Giorgi J, Wang X, Sahar N, Shin WY, Bader GD, Wang B. 2019. End-to-end Named Entity Recognition and Relation Extraction using Pre-trained Language Models. ArXiv191213415 Cs.

Giorgi JM, Bader GD. 2020. Towards reliable named entity recognition in the biomedical domain. Bioinformatics 36:280-286. doi:10.1093/bioinformatics/btz504

Giorgi JM, Bader GD. 2018. Transfer learning for biomedical named entity recognition with neural networks. Bioinforma Oxf Engl 34:4087-4094. doi:10.1093/bioinformatics/bty449 
Giorgi JM, Nitski O, Bader GD, Wang B. 2020. DeCLUTR: Deep Contrastive Learning for Unsupervised Textual Representations. ArXiv200603659 Cs.

Gyori BM, Bachman JA, Subramanian K, Muhlich JL, Galescu L, Sorger PK. 2017. From word models to executable models of signaling networks using automated assembly. Mol Syst Biol 13:954. doi:10.15252/msb.20177651

Hastings J, Owen G, Dekker A, Ennis M, Kale N, Muthukrishnan V, Turner S, Swainston N, Mendes P, Steinbeck C. 2016. ChEBI in 2016: Improved services and an expanding collection of metabolites. Nucleic Acids Res 44:D1214-1219. doi:10.1093/nar/gkv1031

Imker HJ. 2018. 25 Years of Molecular Biology Databases: A Study of Proliferation, Impact, and Maintenance. Front Res Metr Anal 3:18. doi:10.3389/frma.2018.00018

Jassal B, Matthews L, Viteri G, Gong C, Lorente P, Fabregat A, Sidiropoulos K, Cook J, Gillespie M, Haw R, Loney F, May B, Milacic M, Rothfels K, Sevilla C, Shamovsky V, Shorser S, Varusai T, Weiser J, Wu G, Stein L, Hermjakob H, D’Eustachio P. 2020. The reactome pathway knowledgebase. Nucleic Acids Res 48:D498-D503. doi:10.1093/nar/gkz1031

Khatri P, Sirota M, Butte AJ. 2012. Ten years of pathway analysis: current approaches and outstanding challenges. PLoS Comput Biol 8:e1002375. doi:10.1371/journal.pcbi.1002375

Le Novère N, Hucka M, Mi H, Moodie S, Schreiber F, Sorokin A, Demir E, Wegner K, Aladjem MI, Wimalaratne SM, Bergman FT, Gauges R, Ghazal P, Kawaji H, Li L, Matsuoka Y, Villéger A, Boyd SE, Calzone L, Courtot M, Dogrusoz U, Freeman TC, Funahashi A, Ghosh S, Jouraku A, Kim S, Kolpakov F, Luna A, Sahle S, Schmidt E, Watterson S, Wu G, Goryanin I, Kell DB, Sander C, Sauro H, Snoep JL, Kohn K, Kitano H. 2009. The Systems Biology Graphical Notation. Nat Biotechnol 27:735-741. doi:10.1038/nbt.1558

Liechti R, George N, Götz L, El-Gebali S, Chasapi A, Crespo I, Xenarios I, Lemberger T. 2017. SourceData: a semantic platform for curating and searching figures. Nat Methods 14:1021-1022. doi:10.1038/nmeth.4471

Mack SC, Witt H, Piro RM, Gu L, Zuyderduyn S, Stütz AM, Wang X, Gallo M, Garzia L, Zayne K, Zhang X, Ramaswamy V, Jäger N, Jones DTW, Sill M, Pugh TJ, Ryzhova M, Wani KM, Shih DJH, Head R, Remke M, Bailey SD, Zichner T, Faria CC, Barszczyk M, Stark S, Seker-Cin H, Hutter S, Johann P, Bender S, Hovestadt V, Tzaridis T, Dubuc AM, Northcott PA, Peacock J, Bertrand KC, Agnihotri S, Cavalli FMG, Clarke I, Nethery-Brokx K, Creasy CL, Verma SK, Koster J, Wu X, Yao Y, Milde T, Sin-Chan P, Zuccaro J, Lau L, Pereira S, Castelo-Branco P, Hirst M, Marra MA, Roberts SS, Fults D, Massimi L, Cho YJ, Van Meter T, Grajkowska W, Lach B, Kulozik AE, von Deimling A, Witt O, Scherer SW, Fan X, Muraszko KM, Kool M, Pomeroy SL, Gupta N, Phillips J, Huang A, Tabori U, Hawkins C, Malkin D, Kongkham PN, Weiss WA, Jabado N, Rutka JT, Bouffet E, Korbel JO, Lupien M, Aldape KD, Bader GD, Eils R, Lichter P, Dirks PB, Pfister SM, Korshunov A, Taylor MD. 2014. Epigenomic alterations define lethal CIMP-positive ependymomas of infancy. Nature 506:445-450. doi:10.1038/nature13108

Ostaszewski M, Mazein A, Gillespie ME, Kuperstein I, Niarakis A, Hermjakob H, Pico AR, Willighagen EL, Evelo CT, Hasenauer J, Schreiber F, Dräger A, Demir E, Wolkenhauer O, Furlong LI, Barillot E, Dopazo J, Orta-Resendiz A, Messina F, Valencia A, Funahashi A, Kitano H, Auffray C, Balling R, Schneider R. 2020. COVID-19 Disease Map, building a computational repository of SARS-CoV-2 virus-host interaction mechanisms. Sci Data 7:136. doi:10.1038/s41597-020-0477-8 Pratt D, Chen J, Welker D, Rivas R, Pillich R, Rynkov V, Ono K, Miello C, Hicks L, Szalma S, Stojmirovic A, Dobrin R, Braxenthaler M, Kuentzer J, Demchak B, Ideker T. 2015. 
NDEx, the Network Data Exchange. Cell Syst 1:302-305. doi:10.1016/j.cels.2015.10.001

Rodchenkov I, Babur O, Luna A, Aksoy BA, Wong JV, Fong D, Franz M, Siper MC, Cheung M, Wrana M, Mistry H, Mosier L, Dlin J, Wen Q, O'Callaghan C, Li W, Elder G, Smith PT, Dallago C, Cerami E, Gross B, Dogrusoz U, Demir E, Bader GD, Sander C. 2020. Pathway Commons 2019 Update: integration, analysis and exploration of pathway data. Nucleic Acids Res 48:D489-D497. doi:10.1093/nar/gkz946

Santos MA, Faryabi RB, Ergen AV, Day AM, Malhowski A, Canela A, Onozawa M, Lee J-E, Callen E, Gutierrez-Martinez P, Chen H-T, Wong N, Finkel N, Deshpande A, Sharrow S, Rossi DJ, Ito K, Ge K, Aplan PD, Armstrong SA, Nussenzweig A. 2014. DNA-damage-induced differentiation of leukaemic cells as an anti-cancer barrier. Nature 514:107-111. doi:10.1038/nature13483

Seringhaus MR, Gerstein MB. 2007. Publishing perishing? Towards tomorrow's information architecture. BMC Bioinformatics 8:17. doi:10.1186/1471-2105-8-17

Shannon P, Markiel A, Ozier O, Baliga NS, Wang JT, Ramage D, Amin N, Schwikowski B, Ideker T. 2003. Cytoscape: a software environment for integrated models of biomolecular interaction networks. Genome Res 13:2498-2504. doi:10.1101/gr.1239303

Slenter DN, Kutmon M, Hanspers K, Riutta A, Windsor J, Nunes N, Mélius J, Cirillo E, Coort SL, Digles D, Ehrhart F, Giesbertz P, Kalafati M, Martens M, Miller R, Nishida K, Rieswijk L, Waagmeester A, Eijssen LMT, Evelo CT, Pico AR, Willighagen EL. 2018. WikiPathways: a multifaceted pathway database bridging metabolomics to other omics research. Nucleic Acids Res 46:D661-D667. doi:10.1093/nar/gkx1064

Szklarczyk D, Morris JH, Cook H, Kuhn M, Wyder S, Simonovic M, Santos A, Doncheva NT, Roth A, Bork P, Jensen LJ, von Mering C. 2017. The STRING database in 2017: quality-controlled protein-protein association networks, made broadly accessible. Nucleic Acids Res 45:D362-D368. doi:10.1093/nar/gkw937

Todorov PV, Gyori BM, Bachman JA, Sorger PK. 2019. INDRA-IPM: interactive pathway modeling using natural language with automated assembly. Bioinforma Oxf Engl 35:4501-4503. doi:10.1093/bioinformatics/btz289

Valenzuela-Escárcega MA, Babur Ö, Hahn-Powell G, Bell D, Hicks T, Noriega-Atala E, Wang X, Surdeanu M, Demir E, Morrison CT. 2018. Large-scale automated machine reading discovers new cancer-driving mechanisms. Database $J$ Biol Databases Curation 2018. doi:10.1093/database/bay098

van lersel MP, Villéger AC, Czauderna T, Boyd SE, Bergmann FT, Luna A, Demir E, Sorokin A, Dogrusoz U, Matsuoka Y, Funahashi A, Aladjem MI, Mi H, Moodie SL, Kitano H, Le Novère N, Schreiber F. 2012. Software support for SBGN maps: SBGN-ML and LibSBGN. Bioinforma Oxf Engl 28:2016-2021. doi:10.1093/bioinformatics/bts270 Waagmeester A, Stupp G, Burgstaller-Muehlbacher S, Good BM, Griffith M, Griffith OL, Hanspers K, Hermjakob H, Hudson TS, Hybiske K, Keating SM, Manske M, Mayers M, Mietchen D, Mitraka E, Pico AR, Putman T, Riutta A, Queralt-Rosinach N, Schriml LM, Shafee T, Slenter D, Stephan R, Thornton K, Tsueng G, Tu R, Ul-Hasan S, Willighagen E, Wu C, Su Al. 2020. Wikidata as a knowledge graph for the life sciences. elife 9. doi:10.7554/eLife.52614 
406 FIGURES

A

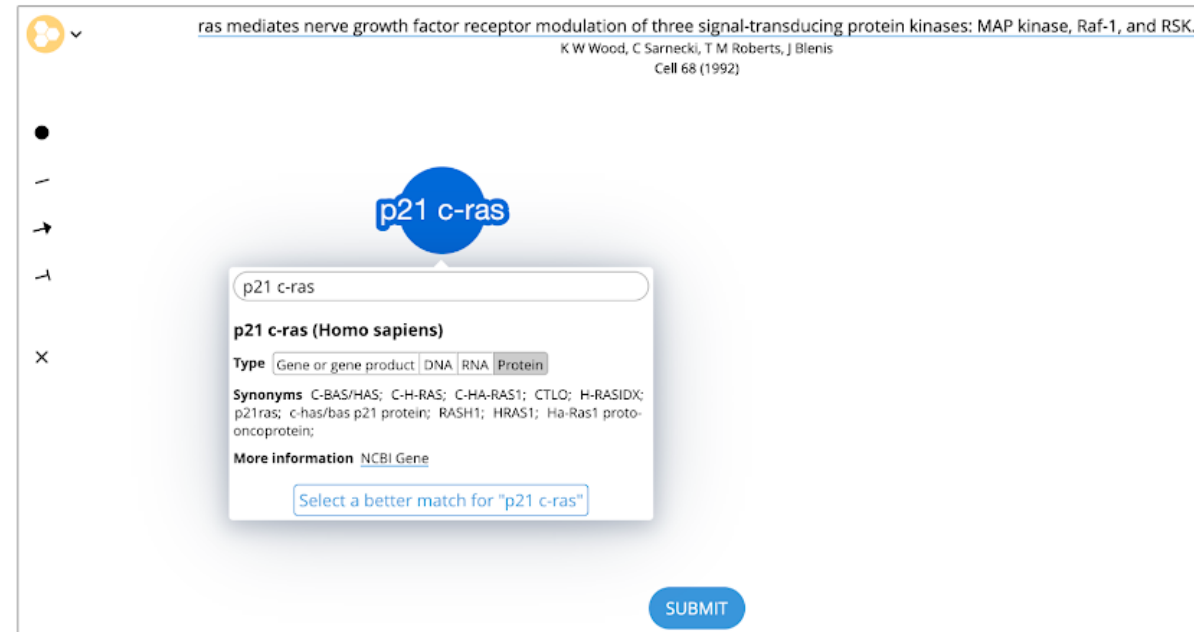

B

407

ras mediates nerve growth factor receptor modulation of three signal-transducing protein kinases: MAP kinase, Raf-1, and RSK. KW Wood, C Sarnecki, TM Roberts, J Blenis

C

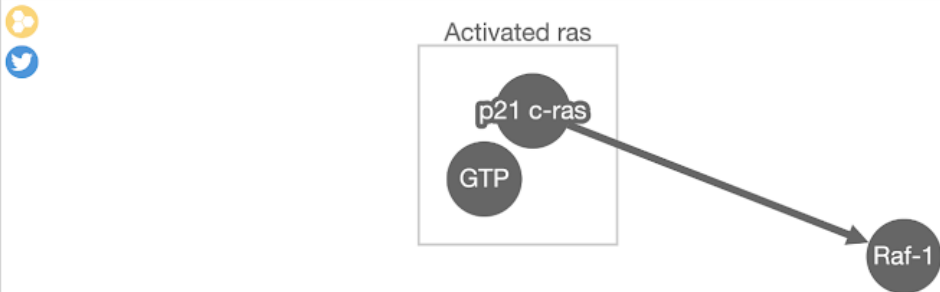

ras mediates nerve growth factor receptor modulation of three signal-transducing protein kinases: MAP kinase, Raf-1, and RSK. KW Wood, C Sarnecki, T M Roberts, J Blenis

Cell 68 (1992) PubMed Google Scholar

Abstract

p21c-ras plays a critical role in mediating tyrosine kinase-stimulated cell growth an differentiation. However, the pathways through which p21c-ras propagates these signals

rernain unknown. We report that in $\mathrm{PC} 12$ cells, expression of a dominant inhibitory mutant of

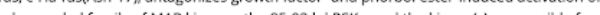
hyperphosphorylation of the proto-oncogene product Raf-1. In addition, we find that

mesce MAP kinases, RSKs, and Raf-1.

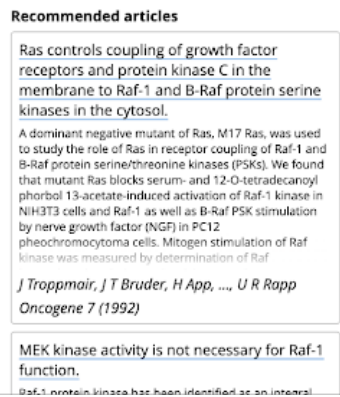

Rheb interacts with Raf- 1 kinase and may function to integrate growth factor-and

protein kinase A-dependent signals,

Rhreb is a recently described member of the Ras farmily
that was originally

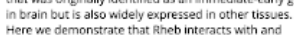
appears to regulate Raf-1 kinase, an essential comm
at the H-Ras sigraling pathwey in direct contrast to Ras, however, the interaction of Rheb with Rat-1 is

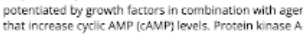
WM Yee PF Worieg Mol. Cell. Biol. 17 (1997) owth factor(aced neuranal differentiation via 
409 Figure 1. The Biofactoid curation tool.

410 Curation in Biofactoid involves creating a visual network representing relationships between 411 genes or molecules. (A) Genes and chemicals are represented by circular shapes (nodes) 412 where users provide a label, the type of gene product, and the organism. A custom search 413 engine matches the label to a corresponding record from a database of genes or chemicals.

414 (B) Relationships are represented by connecting participants with lines, arrows, to indicate 415 activation, or 'T-bars' to represent repression. Users select the mechanism that best 416 describes the interaction. Complexes are represented as participants enclosed by a box, 417 which users can label. (C) Submitted pathways are made publicly available for exploration 418 (browsing) on the Biofactoid website. Users can view information about the article and can 419 access related information, such as other published articles mentioning a particular 420 interaction or participants of an interaction. 